A-paper to be presented at the 26 th annual conven-

tion of the American Institute of Electrical

Engineers, Frontenac, N. Y., June 28, 1909.

Copyright, 1909. By A. I. E. E.

(Subject to final revision for the Transactions.)

\title{
MULTISPEED INDUCTION MOTORS
}

\author{
BY H. G. REIST AND H. MAXWELL
}

The induction motor, as generally used, has a speed which varies slightly with the load but which is usually spoken of as being constant. For work where variable speed is required, a motor with collector rings is used, the speed variation being produced by varying the amount of resistance inserted in the secondary circuit. Such a motor is wasteful of power whenever resistance is used, and the speed varies greatly with the load when much reduction in speed is required.

It sometimes happens that the work is of such a character that two or three speeds will be satisfactory for the operation of the machinery. For such conditions multispeed motors can frequently be used. In these motors the different synchronous speeds are produced by changing the number of poles in the magnetic circuit. Each of these speeds is fixed if no resistance is used in the secondary circuit, so that a change in load will produce only a very slight variation in speed, and the motor will operate efficiently at each speed. With multispeed motors, as with ordinary motors, secondary resistance may be used to vary the speed below each of the synchronous speeds. In this way the complete variation of the speed over a wide range may be obtained more efficiently than with a simple collector-ring motor, but the motor and the control apparatus may be so complicated that the saving of power is not warranted.

A change in the number of poles may be produced in any one of the following ways:

a. By the use of single magnetic and electric circuits, changing the number of poles by re-grouping the coils. 
$b$. By the use of a single magnetic circuit and independent electric circuits.

c. By means of separate magnetic and electric circuits.

The selection and design of the multispeed motor is affected principally by the number of speeds required, the ratio of the desired speeds, and the ratio of the maximum outputs at the different speeds.

a. Single magnetic and electric circuit. This type has probably been used more extensively than any other type of multispeed induction motor, due to its economical use of material all of which is active at all speeds. Except in a special case, a disadvantage of this type of motor is the large number of leads which must be brought out of the winding, and the complicated switching apparatus needed for interconnecting them.

For any ratio of two speeds other than $2: 1$, the ordinary three-phase winding requires 33 leads. For a two-phase winding or a three-phase teaser winding, the winding may be arranged with 16 leads. If more than two speeds are desired, the number of leads will be greatly increased and in general will approximate the number made by bringing out the leads of each coil, thus requiring nearly twice as many leads as there are coils in the machine. In this form of motor the same coils are used for the different arrangements of poles, the relative pitch of the winding depending on the number of poles. Since the economy of a winding rapidly decreases when the pitch of its coil is less than one-half, it is disadvantageous to build motors of this type having a greater change of speed than $2: 1$. On account of the large number of leads required, it is not practicable to use this type of winding on the rotors of induction motors, except with a ratio of $2: 1$ speed, with which arrangement only six collector rings are required. With all other speed-ratios the number of collector rings would become prohibitive, so for these motors a squirrel-cage winding is generally used on the secondary.

When the speed-ratio is $2: 1$, this type of motor can be applied advantageously, and a great many have been built and have been in successful operation for several years. A motor wound in this way is practicable for either a wound type of rotor, since it requires only six collector rings, or for a squirrel-cage rotor, which is more generally used.

There are a number of different methods that may be used in connecting the windings of such motors, the selection of the 
connection in any case depending on the relative maximum outputs required at the two speeds. The connection which has frequently been used where the material is worked to the best advantage, has a half-speed rating of from 60 per cent to 70 per cent of full-speed rating. The following table gives several different connections that may be used, with the approximate relative outputs of the different connections. The third combination shown on this table is the one generally used, as it best suits the average conditions of a load which requires constant torque at the two speeds; that is, when the output of the motor is proportional to the speed.

\begin{tabular}{|c|c|c|}
\hline Speed & Connection & $\begin{array}{c}\text { Approximate } \\
\text { maximum output }\end{array}$ \\
\hline (1) $\begin{array}{r}100 \\
50\end{array}$ & $\begin{array}{l}\text { 2-Circuit Delta } \\
\text { Y-delta }\end{array}$ & $\begin{array}{r}100 \\
11\end{array}$ \\
\hline (2) $\begin{array}{r}100 \\
50\end{array}$ & $\stackrel{2 \text {-circuit } \mathrm{Y}}{\mathrm{Y}}$ & $\begin{array}{r}100 \\
22\end{array}$ \\
\hline (3) 1000 & $\begin{array}{lll}2 & \text { " } & Y \\
1 & \text { Delta }\end{array}$ & $\begin{array}{r}100 \\
66\end{array}$ \\
\hline (4) $\begin{array}{r}1000 \\
50\end{array}$ & $\begin{array}{lll}1 & \text { " Delta } \\
2 & \text { Y }\end{array}$ & $\begin{array}{l}100 \\
117\end{array}$ \\
\hline (.5) 1001 & $\begin{array}{lll}1 & “ & Y \\
2 & \cdots & Y\end{array}$ & $\begin{array}{l}100 \\
350\end{array}$ \\
\hline (6) $\begin{array}{r}100 \\
50\end{array}$ & $\begin{array}{l}\text { Y Delta } \\
2 \text { Circuit Delta }\end{array}$ & $\begin{array}{l}100 \\
700\end{array}$ \\
\hline
\end{tabular}

These values are approximate and will vary slightly with the ratio of the reactance to the resistance and also with the ratio of the coil-end to the slot part of the reactance leakage.

From an inspection of the table it will be seen that there is a wide range of maximum torque from a little over 10 per cent for the half-speed connection, to about seven times that of the full-speed connection. These various combinations are often valuable in extraordinary cases to maintain a good power-factor and efficiency at the desired loads. In most of these combinations the material is not worked to equal advantage at both speeds.

b. Motors with a single magnetic circuit and independent electric circuits. This type of multispeed motor has the advantage of using the same magnetic circuit at all speeds, and therefore requires only one stator and rotor structure, the same as an ordinary motor. These motors, having independent windings, have three leads for each speed in the stator for threephase motors and four for two-phase motors. 
The slots in this type of motor are usually made large enough to contain the two or more windings used in making the several windings for the different speeds. It is theoretically possible to obtain a number of synchronous speeds, but it is usually impracticable to use more than two windings. The relative maximum outputs are independent of each other, but to work the magnetic material to advantage it should be approximately proportional to the speed. The motors of this type have to be built large for their output in order to allow space for the increased amount of copper and insulation. The space occupied by insulation becomes excessive on high-voltage motors so that this type is applicable chiefly to low-voltage windings. It is possible in this form of motor to utilize one or more of the windings mentioned in type $(a)$, thus increasing the possible number of speeds. Since there are two or more distinct windings, the construction is more complicated and the motors are somewhat more difficult to repair than when only one winding is used.

c. Separate magnetic and electric circuits. The concatenated motor is the only motor of this type in general use. This consists of two motors, the rotors of which are mounted on the same shaft arranged so that the secondary of the first motor is directly connected to the primary of the second motor. The two motors may have the same number of poles, but usually the number of poles is different. The effect produced by the second motor is that of adding to or subtracting its poles from the first motor. It will be seen that if the two motors have a different number of poles, there will be four synchronous speeds, two for the motors independently and two for the combination. If the speed corresponding to the difference in the number of poles is higher than the synchronous speed of the first motor, the set will not accelerate by itself to this speed; and although there might be ways of accelerating to this speed, the switching devices will be complicated so that from a practical standpoint this combination is not generally considered.

By using in concatenation two of the half-speed motors mentioned in class $(a)$, the number of speed combinations is very large, and the number of leads and switching of the electric circuits, while complicated, may be warranted in some cases. If, for instance, we take a 4-8 pole and a 6-12-pole motor, we can obtain combinations to give motors having speeds equivalent to every possible number of poles from 4 to 20 , except 18 . 
The ratio of speed of the two motors is limited by the necessity of changing the poles of each motor, two at a time. A further limitation is the centrifugal stresses in the larger motor at high speeds. If the individual motors have the same synchronous speed and can be run in multiple at the high speed, the ratio of the maximum output of the high speed to the low speed is about $4: 1$. If the load requires this ratio of work with the different speeds, the material in the motor is worked to the best advantage.

The two conditions - of having a load as desired in the foregoing and having speeds with ratio of $2: 1$-rarely exist, so in general the material cannot be economically used in concatenated motors. The ratio of the outputs mentioned is usually unfavorable, since in general the output required at the lower speed is more than one-quarter that required at the higher speed. There

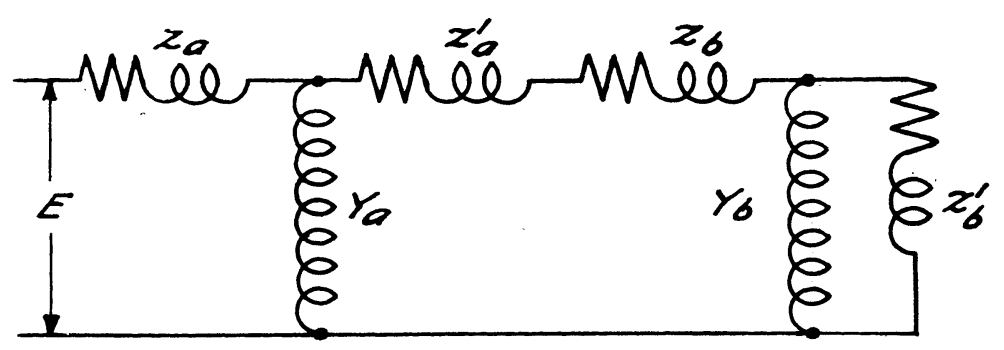

Eis.1

are some classes of work, however, such as the driving of blowers, where approximately this load-ratio may exist.

On account of the large variation in output at the two speeds, the concatenated motors generally use material at the high speed to a very great disadvantage, so that such motors are usually large and expensive for the work to be done. The most favorable condition for the use of the concatenated motor is when the various speeds required are close together. In such a case the two motors would be very different in size and the large motor would be used for the highest speed; for example, if the large motor had 16 poles and the small one had 4 , speeds corresponding to 12,16 , and 20 poles could be obtained.

Another disadvantage of the concatenated motor is its low power-factor. This is inherently low, even at the highest speed, owing to the fact that the normal load carried on the individual 
motors is seldom as large in proportion as the necessary maximum output of these motors in order to obtain sufficient output when the motors are used in combination; in other words, the higher-speed motors will give a poor power-factor because they are underloaded.

To explain the characteristics of the concatenated motors

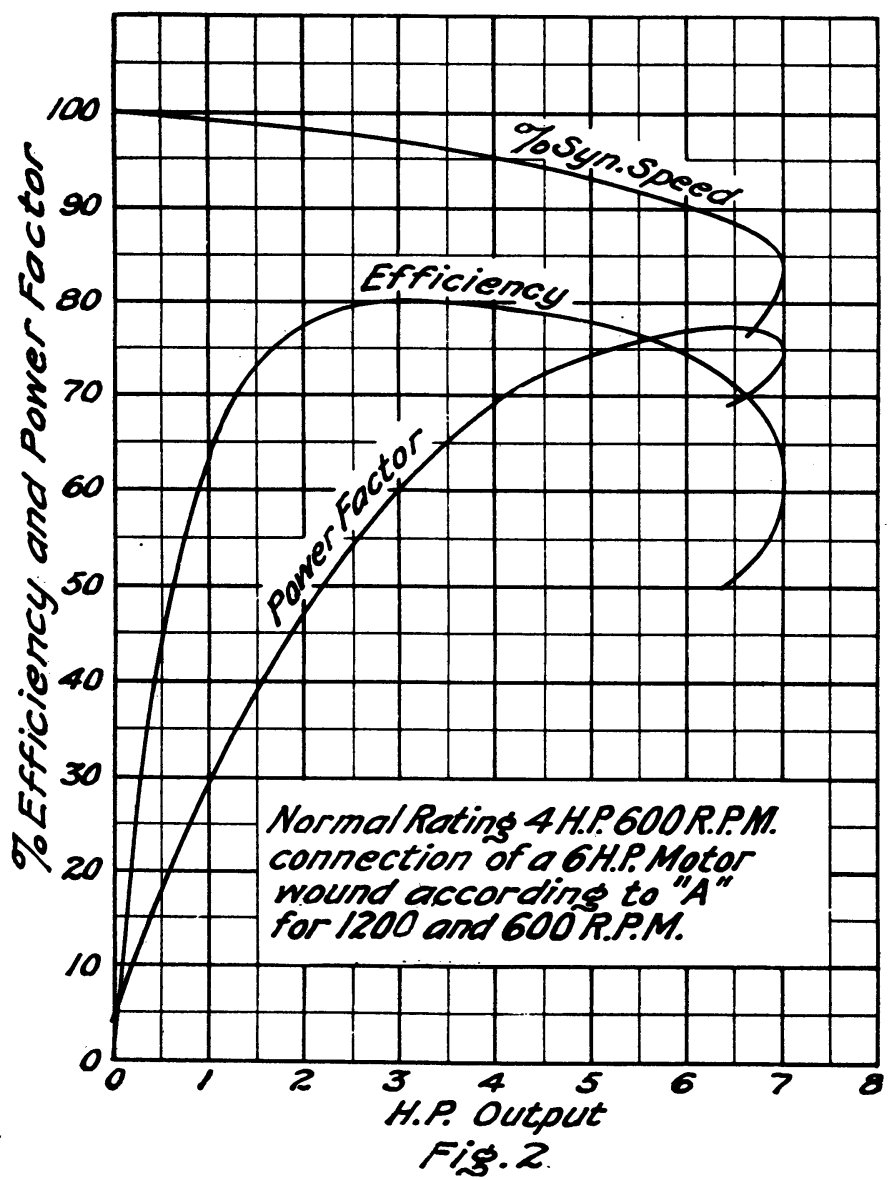

more clearly, attention is called to Fig. $1^{*}$ which represents circuits equivalently arranged;

In this diagram $Z_{a}$ and $Z_{b}$ represent the impedance of the primary circuit of the two motors $A$ and $B$, and $Z_{a}{ }^{\prime}, Z_{b}{ }^{\prime}$ their

* For explanation of Fig. 1 see E. Arnold, "Die Wechselstromtechnik" Vol. 5, Part 1, Fig. 265. 
secondary impedance, while $Y_{a}$ and $Y_{b}$ are their exciting admittances. The resistance component of the impedance is here understood to be corrected for each circuit according to the frequency of that circuit, so that all terms are given as their equivalent value for full frequency.

The load current travels around through $Z_{a}, Z_{a}{ }^{\prime}, Z_{b}, Z_{b}{ }^{\prime}$, in series, while the magnetizing circuits $Y_{a}$ and $Y_{b}$ are across the line with full voltage impressed upon them, except for the voltage drop in the primary circuit in the winding of $A$ for $Y_{n}$,

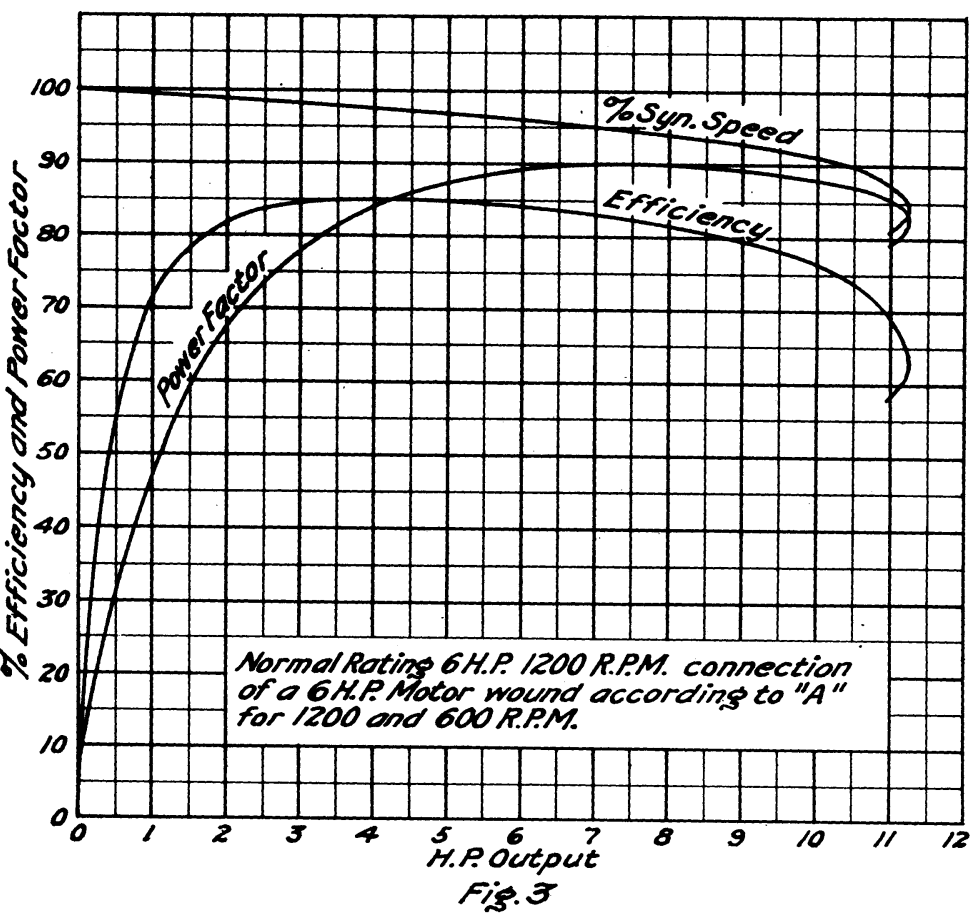

and the additional drop of the secondary of $A$ and the primary of $B$ for $Y_{b}$. Since these voltage-drops are small at normal load, we can neglect them and say that the impedances of both motors are added, and, likewise, that the magnetizing currents of the two are added.

The maximum output of a motor is inversely proportional to its impedance at a given frequency and voltage, so that if - the two single motors have equal impedance, the maximum output of the concatenated couple is approximately one-half 
the maximum output of one motor alone, and its magnetizing current is approximately twice that of one motor-hence its inherent low power-factor in concatenation.

Two curves from actual tests of motors are reproduced to illustrate the points that we have tried to bring out regarding the various characteristics of two of the types of motors mentioned. The curves in Figs. 2 and 3 show the characteristics of a motor of type $(a)$ wound for full speed and half speed ac-

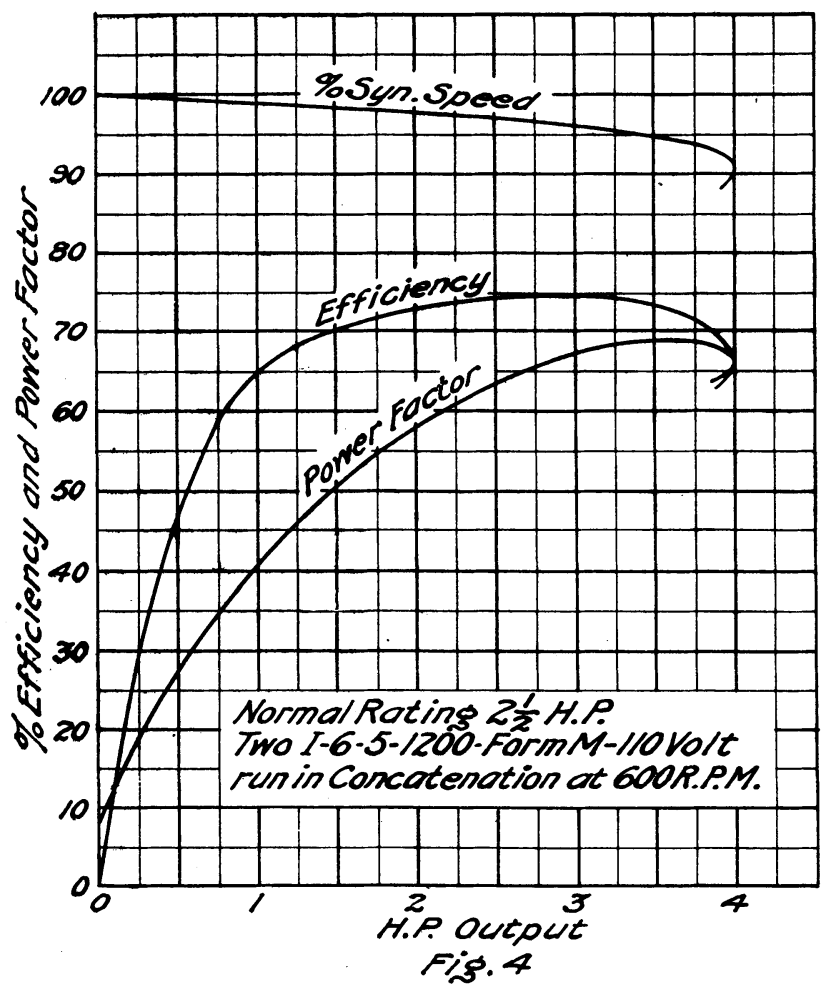

cording to No. :3 connection of the table given. The curves in Fig. 4 show the characteristics of two motors of equal size. run in concatenation. Fig. 5 shows the characteristics of one of the motors in Fig. 4 run at the high-speed connection, the other motor being idle. If both motors were run in multiple at the high speed, the output would be twice as great as indicated in Fig. 5 .

As a suggestion in the selection of the best type for various 
conditions, we have drawn up the following conclusions which must, however, be interpreted very broadly as there are many modifying circumstances.

1. For two speeds having a ratio of $2: 1$, use motor type $(a)$.

2. For two speeds having other ratios than $2: 1$, use motor type $(b)$, except when the winding and insulation require tor much space.

3. When three speeds are required, two having a ratio of $2: 1$, the other being intermediate, type $(b)$ may be used with one winding connected as in $(a)$.

4. If four speeds are necessary, one speed being one-half of

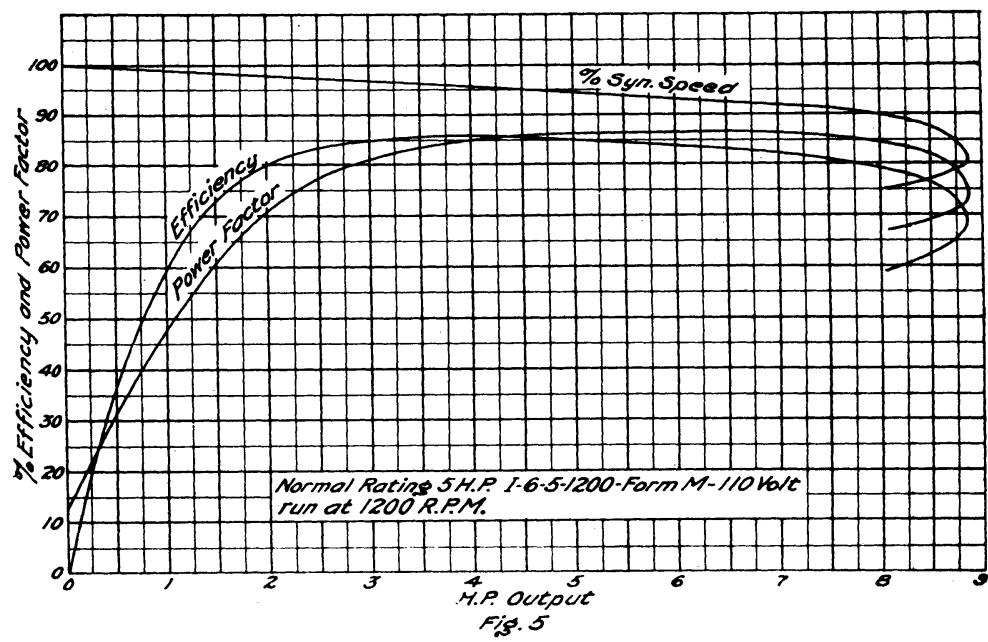

the highest speed, and the lowest speed one-half of the next highest speed, type (b) may be used, with each winding such as is used in type $(a)$.

5. Use type (c) concatenated motors, when two or more speeds close together are required.

6. Use concatenated motors when polar windings on the rotor are necessary with more than two speeds, or if the two speeds differ from $2: 1$.

7. Use concatenated motors for high-voltage machines except when type $(a)$ can be used. 